\title{
Density and correlations of topological objects near the transition temperature in lattice gluodynamics
}

\author{
V. G. Bornyakov $\odot$ \\ Institute for High Energy Physics, NRC “Kurchatov Institute," 142281 Protvino, Russia, \\ Institute of Theoretical and Experimental Physics, NRC “Kurchatov Institute," 117218 Moscow, Russia, \\ School of Biomedicine, Far East Federal University, 690950 Vladivostok, Russia \\ E.-M. Ilgenfritz \\ Joint Institute for Nuclear Research, BLTP, 141980 Dubna, Russia \\ B. V. Martemyanov \\ Institute of Theoretical and Experimental Physics, NRC "Kurchatov Institute," 117218 Moscow, Russia, \\ National Research Nuclear University MEPhI, 115409 Moscow, Russia, \\ Moscow Institute of Physics and Technology, 141700 Dolgoprudny, Moscow Region, Russia
}

(Received 9 January 2020; accepted 3 June 2020; published 30 June 2020)

\begin{abstract}
Topological lumps are known to be present in gluonic fields of $S U(3)$ gluodynamics. Near the transition temperature they were classified either as constituents of nondissociated (anti)calorons, or as constituents of (anti)dyon pairs, or as isolated (anti)dyons. In this paper we study the density and correlation functions of these objects at temperature $T / T_{c}=0.96$.
\end{abstract}

DOI: 10.1103/PhysRevD.101.114510

\section{INTRODUCTION}

In Ref. [1] we have investigated topological objects formed in gluonic fields of $S U(3)$ gluodynamics near the transition temperature. It was done with the help of low lying modes of the overlap Dirac operator. These modes allow us to construct the topological charge density corresponding to the three constituents (dyons) of a caloron with nontrivial holonomy [2-4] when three types of fermionic temporal boundary conditions are used.

The dyons play the decisive role in a recent model of the QCD vacuum proposed by Shuryak and collaborators [5-9]. The density of dyons and their interaction determined in the lattice simulations are important inputs for this model. This motivated us to return to the investigation of $S U$ (3) pure gluodynamics. We will present the results obtained just below the confinement-deconfinement phase transition at $T / T_{c}=0.96$. In particular, we present a numerical value for the dyon number density and compare it with the model prediction [7]. Results for various dyon correlation functions are presented for the first time.

Published by the American Physical Society under the terms of the Creative Commons Attribution 4.0 International license. Further distribution of this work must maintain attribution to the author(s) and the published article's title, journal citation, and DOI. Funded by SCOAP ${ }^{3}$.
We study the density and interaction of dyons using the same method to construct the topological charge density as in Ref. [1]. The new element introduced into this method is the new criterion to determine the number of low lying modes of the overlap Dirac operator that should be used for the construction of the UV-filtered fermionic topological charge density.

In Sec. II, the details of the lattice ensemble created at a temperature near the deconfining transition are described. In Sec. III, we sketch the fermionic construction of the topological charge densities as applied for three types of fermionic temporal boundary conditions. A cluster analysis of the resulting topological charge densities provides us with the possibility to localize dyons of different types. The results for dyon densities and dyon correlation functions are presented and discussed. Finally, we present our conclusions in Sec. IV.

\section{SETUP OF THE INVESTIGATION}

The $S U(3)$ gauge field configurations for this investigation have been generated on a lattice of size $24^{3} \times 6$ by sampling the pure $S U(3)$ gauge theory using the LüscherWeisz action [10].

In addition to the plaquette term (pl), the Lüscher-Weisz action includes a sum over all $2 \times 1$ rectangles (rt) and a sum over all parallelograms (pg), i.e., all possible closed loops of length 6 along the edges of all 3 -cubes, 


$$
\begin{aligned}
S[U]= & \beta\left(\sum_{\mathrm{pl}} \frac{1}{3} \operatorname{Re} \operatorname{Tr}\left[1-U_{\mathrm{pl}}\right]+c_{1} \sum_{r t} \frac{1}{3} \operatorname{Re} \operatorname{Tr}\left[1-U_{\mathrm{rt}}\right]\right. \\
& \left.+c_{2} \sum_{\mathrm{pg}} \frac{1}{3} \operatorname{Re} \operatorname{Tr}\left[1-U_{\mathrm{pg}}\right]\right),
\end{aligned}
$$

where $\beta$ is the principal inverse coupling parameter, while the coefficients $c_{1}$ and $c_{2}$ are computed using the results of the one-loop perturbation theory and tadpole improvement [11-13]:

$$
c_{1}=-\frac{1}{20 u_{0}^{2}}[1+0.4805 \alpha], \quad c_{2}=-\frac{1}{u_{0}^{2}} 0.03325 \alpha .
$$

For a given $\beta$, the tadpole factor $u_{0}$ and the lattice coupling constant $\alpha$ are self-consistently determined in terms of the average plaquette,

$u_{0}=\left(\left\langle\frac{1}{3} \operatorname{Re} \operatorname{Tr} U_{\mathrm{pl}}\right\rangle\right)^{1 / 4}, \quad \alpha=-\frac{\ln \left(\left\langle\frac{1}{3} \operatorname{Re} \operatorname{Tr} U_{\mathrm{pl}}\right\rangle\right)}{3.06839}$,

in the course of a series of iterations.

The ensemble of 100 configurations for the overlap analysis has been generated at $\beta=8.20$. According to previous work [14], this ensemble corresponds to a temperature of $T=287 \mathrm{MeV}$ [15], close to the phase transition temperature $T_{c}=300 \mathrm{MeV}$ [16].

\section{TOPOLOGICAL CLUSTERING}

We have analyzed the configurations of the ensemble by identifying and investigating $N \leq 30$ zero and near-zero eigenmodes of the overlap Dirac operator. The spectral analysis has been performed for three types of temporal boundary conditions (B.C.) applied to the fermion field $\psi$ :

$$
\psi(1 / T)=\exp (i \phi) \psi(0)
$$

with

$$
\phi=\left\{\begin{array}{l}
\phi_{1} \equiv-\pi / 3 \\
\phi_{2} \equiv+\pi / 3 \\
\phi_{3} \equiv \pi
\end{array}\right.
$$

For these three types of B.C.s, the fermionic zero mode is maximally localized at one of the three constituent dyons in the case of a single-caloron solution with maximally nontrivial holonomy. For each of these B.C.s, we have determined the topological index and have checked that it was independent of the choice of $\phi$. The obtained spectral density is also independent of B.C.s and is nonzero for small eigenvalues signaling spontaneous violation of chiral symmetry.
In order to proceed further, we have reconstructed from the zero and nonzero modes the profiles of the UV-filtered topological charge density corresponding to the chosen fermionic boundary condition according to its spectral representation (for details see $[17,18]$ ),

$$
q_{i, N}(x)=-\sum_{j=1}^{N}\left(1-\frac{\lambda_{i, j}}{2}\right) \psi_{i, j}^{\dagger}(x) \gamma_{5} \psi_{i, j}(x),
$$

where $j$ enumerates the eigenvalues $\lambda_{i, j}$ equal and closest to zero. These precise eigenvalues $\lambda_{i, j}$, as well as the corresponding modes $\psi_{i, j}(x)$, are characterized by the $i$ th boundary condition. Correspondingly, the UV-filtered topological density $q_{i, N}(x)$ depends on the boundary condition too.

Note that the average over three fermionic topological charge densities computed for three types of the boundary conditions can be used as a definition of the fermionic topological charge density. It was shown in Ref. [19] that this fermionic topological charge density has strong correlation with the gluon topological charge density computed after some smoothing (the gradient flow was used in [19] as a smoothing procedure).

We have applied a cluster analysis where the minimal (in absolute value) topological density inside all clusters $q_{\text {cut }}$ is variably chosen for each lattice configuration. The cutoff $q_{\text {cut }}$ has been chosen such as to resolve a maximal number of internally connected (while mutually separated) clusters. It has been independently adapted for each configuration. The purpose of the cluster analysis was to discover extended objects that we are going to consider as dyon candidates (of the respective type).

We have found the following average numbers of clusters per configuration (comprising all 100 configurations) corresponding to the three boundary conditions $i=1,2,3$ :

$N_{1,30}=27.0(4), \quad N_{2,30}=27.0(4), \quad N_{3,30}=27.0(4)$,

which completely agree within errors (the latter given in parentheses). Therefore, in the confining (center symmetric) phase the abundance of all three types of clusters is equal, and the clusters can be interpreted as dyons with maximally nontrivial holonomy. For further comparison, we made the same calculations with $N=10$ and $N=20$ low lying modes taken into account in the reconstruction of the topological density:

$$
\begin{aligned}
& N_{1,10}=14.6(3), \quad N_{2,10}=14.2(3), \quad N_{3,10}=14.1(3), \\
& N_{1,20}=21.0(3), \quad N_{2,20}=21.2(4), \quad N_{3,20}=21.0(4) .
\end{aligned}
$$

While the mutual equality of multiplicity remains, the total number of clusters changes monotonically with the number of analyzing modes. 
Taking the lattice spacing value $a=0.115 \mathrm{fm}$ from Ref. [14], we obtain the physical three-dimensional dyon cluster density $\rho=\left(N_{1,30}+N_{2,30}+N_{3,30}\right) /(24 a)^{3}=$ $3.9(6) \mathrm{fm}^{-3}$.

Next, we have checked whether clusters of different types, i.e., clusters determined for three types of the boundary conditions (5), are correlated. To this end, we computed a number of isolated clusters, $N_{d, 30}$, a number of pairs of different type clusters connected to each other, $N_{d d, 30}$, and a number of triplets of connected clusters of different types (full calorons), $N_{d d d, 30}$. The clusters of different types were counted as being connected in pairs or triplets if the distance between them was less than two lattice spacings. We found (on average per configuration),

$N_{d, 30}=40(1), \quad 2 N_{d d, 30}=24.3(7), \quad 3 N_{d d d, 30}=16.7(7)$.

Interpreted in terms of calorons of nontrivial holonomy, this means that we see full caloronlike clusters consisting of three constituents on one hand and also completely dissolved caloron constituents on the other hand.

Again, for further comparison, we made the same calculations with $N=10,20$ low lying modes taken into account:

$$
\begin{aligned}
& N_{d, 10}=22(1), \quad 2 N_{d d, 10}=11.6(4), \quad 3 N_{d d d, 10}=9.1(5), \\
& N_{d, 20}=32.4(9), \quad 2 N_{d d, 20}=17.4(6), \quad 3 N_{d d d, 20}=13.3(7) \text {. }
\end{aligned}
$$

We computed the topological susceptibility $\chi=$ $\left\langle Q^{2}\right\rangle / V_{4}$ (with $Q$ being the topological charge of configuration and $V_{4}$ being its $4 d$ volume) and found $\chi=$ $(187 \pm 6 \mathrm{MeV})^{4}$. This result is in very good agreement with that of Ref. [14], obtained at the same temperature and for the same action as in our work. Our modeling of the topological susceptibility by an ensemble of dyons, dyon pairs, and full calorons gives rise to the expression

$$
\chi_{\text {model }}=Q_{d}^{2} n_{d, N}+Q_{d d}^{2} n_{d d, N}+Q_{d d d}^{2} n_{d d d, N},
$$

where $Q_{d}= \pm 1 / 3, Q_{d d}= \pm 2 / 3$ and $Q_{d d d}= \pm 1, n_{d, N}=$ $N_{d, N} / V_{4}, n_{d d, N}=N_{d d, N} / V_{4}, n_{d d d, N}=N_{d d d, N} / V_{4}$ are the densities of isolated dyons, dyon pairs, and full calorons, respectively. We found $\chi_{\text {model }}=(169 \pm 2 \mathrm{MeV})^{4},(187 \pm$ $2 \mathrm{MeV})^{4}$ and $(201 \pm 2 \mathrm{MeV})^{4}$ for $N=10,20,30$, respectively. This is an estimate of the topological susceptibility for uncorrelated "objects" [20] of the respective charge.

As one can see, the best agreement of our modeling with our own results and the result of Ref. [14] is obtained for $N=20$ modes of the overlap operator. We take this agreement as a criterion for choosing the number of modes $N$, and we further use exclusively $N=20$ modes of the overlap operator in calculations of correlation functions. The three-dimensional dyon cluster density in this case is equal to $\rho=3.03 \mathrm{fm}^{-3}$. Note that the respective dimensionless value $\rho / T^{3}=0.98$ should be compared with the value 0.74 obtained in Ref. [7] for the density of dyons in the case of the $\mathrm{SU}(2)$ dyon model at a temperature close to $T_{c}$.

For each configuration, we calculated the distances between topological lump objects of different types (dyons/antidyons of three types): two dyons (or antidyons) of the same type $\left(d_{i} d_{i}\right)$, a dyon and an antidyon of the same type $\left(d_{i} \bar{d}_{i}\right)$, two dyons (or antidyons) of different types $\left(d_{i} d_{j}\right)$, and a dyon and antidyon of different types $\left(d_{i} \bar{d}_{j}\right)$. The numbers of these pairs in bins of (three-dimensional) distances from $x$ to $x+d x$ divided by the numbers of lattice points falling in the same bins are presented as functions of the calculated distances over the range from zero distance to the maximal distance $\sqrt{(24 / 2)^{2}+(24 / 2)^{2}+(24 / 2)^{2}} \approx$ 20 lattice units. Normalized (to the total density of dyons and antidyons of all types squared), the correlators of dyon and antidyon densities,

$$
\begin{gathered}
\left\langle d_{i} d_{i}(x)\right\rangle \equiv \frac{\sum_{i}\left\langle d_{i}(x) d_{i}(0)+\bar{d}_{i}(x) \bar{d}_{i}(0)\right\rangle}{\left(\sum_{i}\left\langle d_{i}+\bar{d}_{i}\right\rangle\right)^{2}}, \\
\left\langle d_{i} \bar{d}_{i}(x)\right\rangle \equiv \frac{\sum_{i}\left\langle d_{i}(x) \bar{d}_{i}(0)+\bar{d}_{i}(x) d_{i}(0)\right\rangle}{\left(\sum_{i}\left\langle d_{i}+\bar{d}_{i}\right\rangle\right)^{2}}, \\
\left\langle d_{i} d_{j}(x)\right\rangle \equiv \frac{\sum_{i \neq j}\left\langle d_{i}(x) d_{j}(0)+\bar{d}_{i}(x) \bar{d}_{j}(0)\right\rangle}{\left(\sum_{i}\left\langle d_{i}+\bar{d}_{i}\right\rangle\right)^{2}}, \\
\left\langle d_{i} \bar{d}_{j}(x)\right\rangle \equiv \frac{\sum_{i \neq j}\left\langle d_{i}(x) \bar{d}_{j}(0)+\bar{d}_{i}(x) d_{j}(0)\right\rangle}{\left(\sum_{i}\left\langle d_{i}+\bar{d}_{i}\right\rangle\right)^{2}},
\end{gathered}
$$

are shown in Fig. 1 as histograms with bins of one lattice spacing size. The errors are shown in the centers of the corresponding bins.

In Fig. 1(c), the first two bins are not shown. Respective correlator values are equal to 51.6 and 4.47 for the first and second bins, correspondingly. We see that two dyons (or antidyons) of different types are attracting (positively correlated) at small distances. At this point we are in agreement with the model results of Ref. [7]. As a result of this attraction, half of the dyons and antidyons are combined in dyon pairs and dyon triplets (full calorons). In Fig. 1(a), one can also see some attraction at small distances for two dyons (or antidyons) of the same type (which cannot form dyon pairs and full calorons). This is in contrast to the repulsion postulated in Ref. [7]. As for dyon and antidyon interaction, we observe a repulsion at small distances of strength independent of the types of dyons and antidyons [see Figs. 1(b) and 1(d)]. At larger distances, in all four cases, we do not see any interaction (nontrivial correlation). We note that our results for correlators (8)-(11) computed with $N=10$ and 30 are qualitatively the same as shown in Fig. 1. 

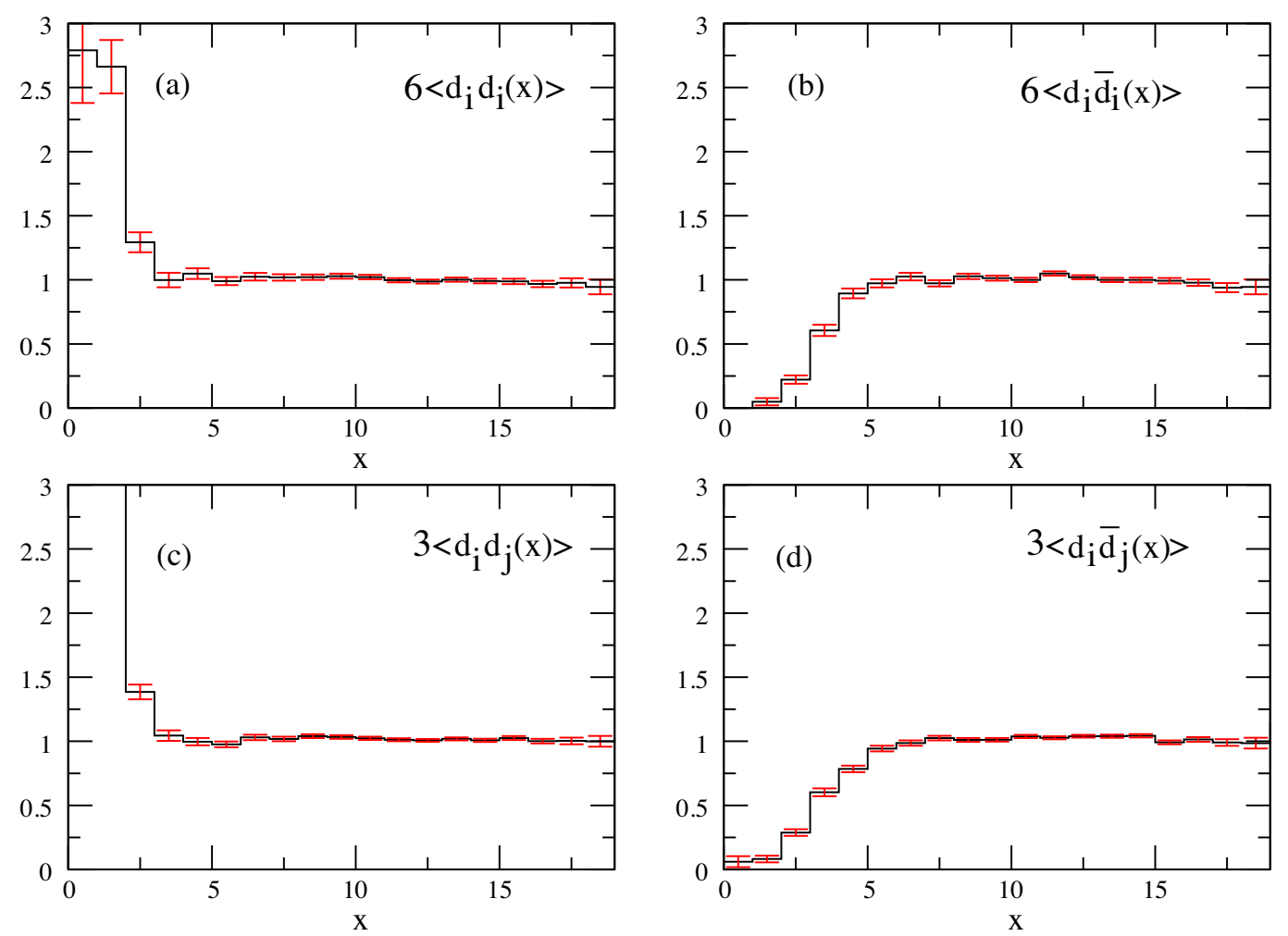

FIG. 1. Correlators (normalized to the total density of dyons and antidyons of all types squared) of dyon and antidyon densities are shown as histograms over distance with bins of one lattice spacing: (a) dyons (or antidyons) of the same type; (b) dyon and antidyon of the same type; (c) dyons (or antidyons) of different types; (d) dyon and antidyon of different types. The errors are shown in the centers of the corresponding bins.

\section{CONCLUSIONS}

In $S U$ (3) lattice gauge theory, using a small number of modes of the overlap Dirac operator with eigenvalues closest to zero, we have investigated the clusters formed by the UVfiltered fermionic topological charge density. The topological charge density has been computed for three different types of temporal boundary conditions applied to the overlap Dirac operator. Assuming that these clusters correspond to dyons, we have obtained their frequency of occurrence and demonstrated the tendency to combine into triplets (calorons) or to form pairs of dyons apart from the remaining isolated dyons.

We accomplished a first lattice computation of the dyon correlation functions defined in Eqs. (8)-(11). We found, at small distances, attraction for two dyons (or antidyons) and repulsion for dyons and antidyons. The attraction for two dyons (or antidyons) of different types is larger than the attraction for two dyons (or antidyons) of the same type. Repulsion for dyons and antidyons does not depend on the types of dyons and antidyons. At larger distances, in all cases, we do not see any correlations.

\section{ACKNOWLEDGMENTS}

The work was completed due to support of the Russian Foundation for Basic Research via Grant No. 18-0240130 mega.
[1] E.-M. Ilgenfritz, B. Martemyanov, and M. Müller-Preussker, Phys. Rev. D 89, 054503 (2014).

[2] T. C. Kraan and P. van Baal, Nucl. Phys. B533, 627 (1998).

[3] T. C. Kraan and P. van Baal, Phys. Lett. B 435, 389 (1998).

[4] K.-M. Lee and C.-H. Lu, Phys. Rev. D 58, 025011 (1998).

[5] E. Shuryak, J. Phys. G 39, 054001 (2012).
[6] P. Faccioli and E. Shuryak, Phys. Rev. D 87, 074009 (2013).

[7] R. Larsen and E. Shuryak, Phys. Rev. D 92, 094022 (2015).

[8] R. Larsen and E. Shuryak, Nucl. Phys. A950, 110 (2016).

[9] R. Larsen and E. Shuryak, Phys. Rev. D 96, 034508 (2017).

[10] M. Lüscher and P. Weisz, Commun. Math. Phys. 97, 59 (1985). 
[11] M. Lüscher and P. Weisz, Phys. Lett. 158B, 250 (1985).

[12] J. R. Snippe, Nucl. Phys. B498, 347 (1997).

[13] G. P. Lepage and P. B. Mackenzie, Phys. Rev. D 48, 2250 (1993).

[14] C. Gattringer, R. Hoffmann, and S. Schaefer, Phys. Lett. B 535, 358 (2002).

[15] The scale was fixed by setting the Sommer parameter to $r_{0}=0.5 \mathrm{fm}$.

[16] C. Gattringer, P. E. L. Rakow, A. Schäfer, and W. Söldner, Phys. Rev. D 66, 054502 (2002).
[17] P. Hasenfratz, V. Laliena, and F. Niedermayer, Phys. Lett. B 427, 125 (1998).

[18] E.-M. Ilgenfritz, K. Koller, Y. Koma, G. Schierholz, T. Streuer, and V. Weinberg, Phys. Rev. D 76, 034506 (2007).

[19] V. G. Bornyakov, E.-M. Ilgenfritz, B. V. Martemyanov, and M. Muller-Preussker, Phys. Rev. D 93, 074508 (2016).

[20] This means that the average charges are equal to zero independently for isolated clusters, pairs of connected clusters, and triplets of connected clusters. 